\title{
Identification of areas of emergence of critical flow regimes of nonlinear viscous liquids in a tubular reactor
}

\author{
$S A$ Livshits ${ }^{1, *}, N A$ Yudina $^{1}, T U$ Dunaeva $^{1}, E K$ Nikolaeva $^{1}$ and $S R$ Enikeeva $^{2}$ \\ ${ }^{1}$ Kazan State Power Engineering University, Krasnoselskaya, 51, Kazan, 420034, Russia \\ ${ }^{2}$ Kazan Research Technological University, K. Marksa, 68, Kazan, 420015, Russia
}

\begin{abstract}
The mathematical model describing heat-mass transfer in rheologically complex media in a tubular reactor is developed in the article. Using the example of the continuous production scheme for grade B2 polymethacrylate, solutions of the characteristic equations confirming the possibility of emergence the critical flow regimes for certain sets of control parameters were obtained.
\end{abstract}

\section{Introduction}

In 1928, academician N.N. Semenov published a paper describing a phenomenon later called "thermal explosion in gas media". In the 30s - 40s of the XX century a great development in the study of this subject was made by such prominent scientists as: O.M. Todes, D.A. FrankKamenetsky and Ya.B. Zeldovich.

In 1965, Bostanjinyan S.A., Merzhanov A.G. and Khudyaev S.I. [1] obtained equations similar to the equations proposed by Semenov, but for the flow of a viscous Newtonian fluid. The later investigations of flow of viscous fluids do not give an exhaustive answer to the question of the occurrence of conditions under which a progressive increase in temperature occurs [2-4].

In the mathematical models available to date, the non-Newtonian properties of liquids are not taken into account and there are no methods for calculating the qualitative characteristics using the molecular mass distribution [5-6].

In connection with the need to solve various application tasks arising during design and operation of thermal power plants, the task of theoretical research of heat and mass transfer processes in the laminar flow of Newtonian, dilatant and pseudoplastic chemically reacting liquids in pipes and channels has come to the fore [7-9].

\section{Problem statement}

In this paper, the flow of rheologically complex media [10-11] in a tubular reactor is considered. A closed mathematical model describing heat and mass transfer in the course of fluid motion inside a tubular reactor is developed, which describes the mutual influence of hydrodynamic, heat-mass exchange, rheological and macrokinetics parameters on the polymerization process.

The B2 polymethacrylate is considered as a working agent (monomer) in the reactor, which releases heat as a result of its absolute mixing with the initiator.

While constructing the mathematical model, the following assumptions were made:

1. The flow of the reacting system is laminar, stationary, with the formed profile of the velocity vector at the inlet to the reactor;

2. Mass forces enter the motion equations implicitly, but through excess pressure;

3. The transfer of heat and mass along the direction of the main motion due to thermal conductivity is small in comparison with the forced transfer in the same direction;

4. The thermophysical system characteristics and the coefficients of mutual diffusion change insignificantly during the reaction;

5. The hydrodynamic relaxation time is much shorter than the thermal time, i.e. the velocity vector profile almost instantly adapts to the change in the temperature field;

6. The system under investigation does not possess elastic properties.

For this research, the system of equations of motion, energy conservation, the continuity equation and the mass transfer equations recorded for the monomer and initiator were used [12]. A power-law model [13] was chosen a rheological model.

The system of equations under study:

\footnotetext{
Corresponding author: semen19772004@mail.ru
} 


$$
\begin{gathered}
\rho\left(\frac{\partial V_{r}}{\partial r} V_{r}+\frac{\partial V_{r}}{\partial z} V_{z}\right)=2 \frac{\partial}{\partial r}\left(\mu \frac{\partial V_{r}}{\partial r}\right)-\frac{\partial P}{\partial r}+\frac{\partial}{\partial z}\left(\mu \frac{\partial V_{r}}{\partial z}\right)+\frac{\partial}{\partial z}\left(\mu \frac{\partial V_{z}}{\partial r}\right)-\frac{2 \mu V_{r}}{r^{2}}+\frac{2 \mu}{r} \frac{\partial V_{r}}{\partial z} \\
\rho\left(\frac{\partial V_{z}}{\partial r} V_{r}+\frac{\partial V_{z}}{\partial z} V_{z}\right)=\frac{\partial}{\partial r}\left(\mu \frac{\partial V_{r}}{\partial z}\right)+\frac{\partial}{\partial r}\left(\mu \frac{\partial V_{z}}{\partial r}\right)+2 \frac{\partial}{\partial z}\left(\mu \frac{\partial V_{z}}{\partial z}\right)-\frac{\partial P}{\partial z}+\frac{\mu}{r} \frac{\partial V_{r}}{\partial z}+\frac{\mu}{r} \frac{\partial V_{z}}{\partial r} \\
\frac{\partial V_{r}}{\partial r}+\frac{V_{r}}{r}+\frac{\partial V_{z}}{\partial z}=0 \\
V_{r} \frac{\partial T}{\partial r}+V_{z} \frac{\partial T}{\partial z}=\alpha\left(\frac{\partial^{2} T}{\partial r^{2}}+\frac{1}{r} \frac{\partial T}{\partial r}\right)+\frac{Q_{0} k_{0}}{c_{m}} e^{-E / R T} M^{m} J^{n}+\frac{2 \mu I_{2}}{\rho c_{p}} \\
V_{r} \frac{\partial M}{\partial r}+V_{z} \frac{\partial M}{\partial z}=D_{m}\left(\frac{\partial^{2} M}{\partial r^{2}}+\frac{1}{r} \frac{\partial M}{\partial r}\right)^{-k_{0} e^{-E / R T} M^{m} J^{n}} \\
V_{r} \frac{\partial J}{\partial r}+V_{z} \frac{\partial J}{\partial z}=D_{j}\left(\frac{\partial^{2} J}{\partial r^{2}}+\frac{1}{r} \frac{\partial J}{\partial r}\right)-k_{j 0} e^{-E / R T} J^{s} \\
I_{2}=\left(\frac{\partial V_{r}}{\partial r}\right)^{2}+\frac{1}{2}\left(\frac{\partial V_{r}}{\partial z}+\frac{\partial V_{z}}{\partial r}\right)^{2}+\left(\frac{V_{r}}{r}\right)^{2}+\left(\frac{\partial V_{z}}{\partial z}\right)^{2}
\end{gathered}
$$

Not considering $\frac{\partial \mathrm{P}}{\partial \mathrm{r}}$ and taking into account $Q=\iint_{\Omega} V_{z} d \Omega=\int_{0}^{2 \pi} \int_{0}^{r_{1}} V_{z} r d \phi d r=2 \pi \int_{0}^{r_{1}} V_{z} r \mathrm{dr}$, we have: $\frac{\partial P}{\partial z}=\frac{2 \pi}{Q} \int_{0}^{r_{1}} r\left\{2 V_{z} \frac{\partial}{\partial z}\left(\mu \frac{\partial V_{z}}{\partial z}\right)-\mu\left[\frac{\partial V_{z}}{\partial r} \frac{\partial V_{r}}{\partial z}+\left(\frac{\partial V_{z}}{\partial r}\right)^{2}\right]\right\} d r$
Thus, in order to study the flow of a rheologically complex liquid in a tubular reactor, taking into account the Stokes assumption, we have the following system of equations:

$$
\left\{\begin{array}{l}
\frac{2 \pi}{Q} \int_{0}^{r_{1}} r\left\{2 V_{z} \frac{\partial}{\partial z}\left(\mu \frac{\partial V_{z}}{\partial z}\right)-\mu\left[\frac{\partial V_{z}}{\partial r} \frac{\partial V_{r}}{\partial z}+\left(\frac{\partial V_{z}}{\partial r}\right)^{2}\right]\right\} d r=\frac{1}{r} \frac{\partial}{\partial r}\left(r \mu\left(\frac{\partial V_{r}}{\partial z}+\frac{\partial V_{z}}{\partial r}\right)\right)+2 \frac{\partial}{\partial z}\left(\mu \frac{\partial V_{z}}{\partial z}\right) \\
\frac{\partial V_{r}}{\partial r}+\frac{V_{r}}{r}+\frac{\partial V_{z}}{\partial z}=0 \\
V_{r} \frac{\partial T}{\partial r}+V_{z} \frac{\partial T}{\partial z}=\alpha\left(\frac{\partial^{2} T}{\partial r^{2}}+\frac{1}{r} \frac{\partial T}{\partial r}\right)+\frac{Q_{0} k_{0}}{c_{m}} e^{-E / R T} M^{m} J^{n}+\frac{2 \mu I_{2}}{\rho c_{p}} \\
V_{r} \frac{\partial M}{\partial r}+V_{z} \frac{\partial M}{\partial z}=D_{m}\left(\frac{\partial^{2} M}{\partial r^{2}}+\frac{1}{r} \frac{\partial M}{\partial r}\right)-k_{0} e^{-E / R T} M^{m} J^{n} \\
V_{r} \frac{\partial J}{\partial r}+V_{z} \frac{\partial J}{\partial z}=D_{j}\left(\frac{\partial^{2} J}{\partial r^{2}}+\frac{1}{r} \frac{\partial J}{\partial r}\right)^{-k_{j 0}} e^{-E / R T} J^{s} \\
I_{2}=\left(\frac{\partial V_{r}}{\partial r}\right)^{2}+\frac{1}{2}\left(\frac{\partial V_{r}}{\partial z}+\frac{\partial V_{z}}{\partial r}\right)^{2}+\left(\frac{V_{r}}{r}\right)^{2}+\left(\frac{\partial V_{z}}{\partial z}\right)^{2}
\end{array}\right.
$$


We turn to new, dimensionless functions, variables and parameters [14], i.e.:

$x=\frac{r}{r_{1}}, \dot{z}=\frac{z}{L}$ are the dimensionless functions of coordinates;

$$
\theta=\frac{E}{R T_{0}{ }^{2}}\left(T-T_{0}\right) \text { is the dimensionless function of }
$$
temperature;

$$
\dot{M}=\frac{M}{\widetilde{M}} ; \dot{J}=\frac{J}{\tilde{J}} \text { are the dimensionless and normalized }
$$
concentrations of monomer and initiator;

$$
\alpha=\frac{B}{E} ; \beta=\frac{R T_{0}}{E},
$$

Also we consider the power model as a rheological model:

$$
\mu=\mu_{0} I_{2}^{l}=A \cdot e^{B / R T} \cdot I_{2}^{l}
$$

And so we obtain:

$$
\begin{aligned}
& \int \frac{2 \pi^{2} r_{1}^{4}}{Q^{2}} \int_{0}^{1} x\left\{2 \dot{V}_{z} \frac{\partial}{\partial \dot{z}}\left(e^{-\frac{\alpha \theta}{\beta \theta+1}} \cdot \dot{I}_{2}^{l} \cdot \frac{\partial \dot{V}_{z}}{\partial \dot{z}}\right)-e^{-\frac{\alpha \theta}{\beta \theta+1}} \cdot \dot{I}_{2}^{l} \cdot\left[\frac{\partial \dot{V}_{z}}{\partial x} \frac{\partial \dot{V}_{r}}{\partial \dot{z}}+\left(\frac{\partial \dot{V}_{z}}{\partial x}\right)^{2}\right]\right\} d x= \\
& =\frac{1}{x} \frac{\partial}{\partial x}\left(e^{-\frac{\alpha \theta}{\beta \theta+1}} \cdot \dot{I}_{2}^{l} \cdot x\left(\frac{\partial \dot{V}_{r}}{\partial \dot{z}}+\frac{\partial \dot{V}_{z}}{\partial x}\right)\right)+2 \frac{\partial}{\partial \dot{z}}\left(e^{-\frac{\alpha \theta}{\beta \theta+1}} \cdot \dot{I}_{2}^{l} \cdot \frac{\partial \dot{V}_{z}}{\partial \dot{z}}\right) \\
& \frac{\partial \dot{V}_{r}}{\partial x}+\frac{\dot{V}_{r}}{x}+\frac{\partial \dot{V}_{z}}{\partial \dot{z}}=0 \\
& \left\{\frac{\pi r_{1} R T_{0}^{2}}{Q E}\left(\dot{V}_{r} \frac{\partial \theta}{\partial x}+\dot{V}_{z} \frac{\partial \theta}{\partial \dot{z}}\right)=\alpha \frac{R T_{0}^{2}}{E r_{1}^{2}}\left(\frac{\partial^{2} \theta}{\partial x^{2}}+\frac{1}{x} \frac{\partial \theta}{\partial x}\right)+\frac{\tilde{M}^{m} \tilde{J}^{n} Q_{0} k_{0}}{c_{m}} e^{-E / R T_{0}} e^{\frac{\theta}{\beta \theta++1}} \dot{M}^{m} \dot{J}^{n}+\right. \\
& +\frac{2 A e^{\frac{B}{R T_{0}}} \pi^{2} r_{1}^{4} \mu I_{2}}{Q^{4} \rho c_{p}} e^{-\frac{\alpha \theta}{\beta \theta+1}} \cdot \dot{I}_{2}^{l+1} \\
& \frac{\tilde{M} \pi r_{1}}{Q}\left(\dot{V}_{r} \frac{\partial \dot{M}}{\partial x}+\dot{V}_{z} \frac{\partial \dot{M}}{\partial \dot{z}}\right)=\frac{D_{m} \tilde{M}}{r_{1}^{2}}\left(\frac{\partial^{2} \dot{M}}{\partial x^{2}}+\frac{1}{x} \frac{\partial \dot{M}}{\partial x}\right)-k_{0} e^{-E / R T_{0}} e^{\frac{\theta}{\beta \theta+1}} \dot{M}^{m} \dot{J}^{n} \\
& \frac{\tilde{J} \pi r_{1}}{Q}\left(\dot{V}_{r} \frac{\partial \dot{J}}{\partial x}+\dot{V}_{z} \frac{\partial \dot{J}}{\partial \dot{z}}\right)=\frac{D_{j} \tilde{J}}{r_{1}^{2}}\left(\frac{\partial^{2} \dot{J}}{\partial x^{2}}+\frac{1}{x} \frac{\partial \dot{J}}{\partial x}\right)-k_{j 0} e^{-E / R T_{0}} e^{\frac{\theta}{\beta \theta+1} \dot{J}^{s}} \\
& \dot{I}_{2}=\left(\frac{\partial \dot{V}_{r}}{\partial x}\right)^{2}+\frac{1}{2}\left(\frac{\partial \dot{V}_{r}}{\partial \dot{z}}+\frac{\partial \dot{V}_{z}}{\partial x}\right)^{2}+\left(\frac{\dot{V}_{r}}{x}\right)^{2}+\left(\frac{\partial \dot{V}_{z}}{\partial \dot{z}}\right)^{2}
\end{aligned}
$$

$\dot{V}_{z}=\frac{\pi r_{1}^{2}}{Q} V_{z} ; \dot{V}_{r}=\frac{L \pi r_{1}}{Q} V_{r}$ are the dimensionless

components of the velocity function.

\section{Analytical study of the task}

Taking into account that the functions $\theta, \dot{M}, \dot{J}, \dot{V}_{z}$ are even relatively to $\mathrm{X}$, and that $\dot{\mathrm{V}}_{\mathrm{r}}$ is the odd function, we decompose all functions under investigation into Taylor series in the vicinity of a point $\left(0, \mathrm{z}_{0}\right)$ on the channel axis.

The coefficients in the expansion of the function $\dot{\mathrm{V}}_{\mathrm{r}}$ are found from the continuity equation. Functions $\dot{I}_{2}^{l}$ $\left(\boldsymbol{l}=-\frac{1}{k}\right.$ for polymers), $\boldsymbol{e}^{\frac{\theta}{\beta \theta+1}}$ and $\boldsymbol{e}^{-\frac{\alpha \theta}{\beta \theta+1}}$ are decomposed into Taylor series, and the function $\dot{\mathrm{I}}_{2}$ is not decomposed into a series (its components decompose into series) [15].
Substituting the expansion functions for the investigated functions, we obtain a system of four algebraic equations (the continuity equation is not used, because with its help the coefficients of the Taylor series for the function $\dot{\mathrm{V}}_{\mathrm{r}}$ are obtained) [6].

From consideration of coefficients for the free term and at $\left(\dot{\mathrm{z}}-\mathrm{z}_{0}\right)$, adding initial conditions for the temperature, velocity, concentration of the monomer and initiator, we obtain a system of 16 algebraic equations ( $\mathrm{k}=3, \mathrm{~s}=1, \mathrm{~m}=1, \mathrm{n}=1$ ).

If we substitute the boundary conditions of the first kind and renumber it $\theta_{0}$ for $\mathrm{h}$, we obtain the following characteristic equation for determining the temperature of the working agent: 


$$
\begin{aligned}
& \theta_{1}=\mathrm{h}-\frac{\delta \operatorname{Exp}(\mathrm{h} /(1+\beta \mathrm{h}))}{4}+\frac{1}{16}\left[-\chi \operatorname{Exp}(\alpha \mathrm{h} /(1+\beta \mathrm{h}))+\frac{\delta^{2} \operatorname{Exp}(2 \mathrm{~h} /(1+\beta \mathrm{h})}{4(\beta \mathrm{h}+1)^{2}}\right]- \\
& -\frac{1}{36}\left\{\chi \operatorname{Exp}(\alpha \mathrm{h} /(1+\beta \mathrm{h}))\left[\left(\mathrm{c}_{0}-1\right)\left(1-\operatorname{Exp}\left(-\mathrm{c}_{1}\right)\right)-\frac{\alpha \delta \operatorname{Exp}(\mathrm{h} /(1+\beta \mathrm{h}))}{4(\beta \mathrm{h}+1)^{2}}\right]+\right. \\
& \left.+\frac{3 \delta^{3} \operatorname{Exp}(3 \mathrm{~h} /(1+\beta \mathrm{h}))}{64(1+\beta \mathrm{h})^{4}}-\frac{\beta \delta^{3} \operatorname{Exp}(3 \mathrm{~h} /(1+\beta \mathrm{h}))}{16(1+\beta \mathrm{h})^{3}}-\frac{\chi \delta \operatorname{Exp}((\alpha+1) \mathrm{h} /(1+\beta \mathrm{h}))}{16(1+\beta \mathrm{h})^{2}}\right\}
\end{aligned}
$$

In case when thermal boundary conditions of the third kind are given on the boundary, then, after redesignation $\theta_{0}$ for $\mathrm{h}$, we obtain the following characteristic equation for determining the temperature of the working agent:

$$
\begin{aligned}
& 144 \cdot\left(\mathrm{h}-\theta_{1}\right)=4 \cdot\left\{\chi \cdot \operatorname{Exp}(\alpha \mathrm{h} /(1+\beta \mathrm{h})) \cdot\left(\mathrm{c}_{0}-1\right) \cdot\left(1-\operatorname{Exp}\left(-\mathrm{c}_{1}\right)\right)-\frac{\alpha \cdot \delta \cdot \operatorname{Exp}(\mathrm{h} /(1+\beta \mathrm{h}))}{4(1+\beta \mathrm{h})^{2}}\right]+ \\
& +\frac{3 \delta^{3} \operatorname{Exp}(3 \mathrm{~h} /(1+\beta \mathrm{h}))}{64(1+\beta \mathrm{h})^{4}}-\frac{\beta \delta^{3} \operatorname{Exp}(3 \mathrm{~h} /(1+\beta \mathrm{h}))}{16(1+\beta \mathrm{h})^{3}}-\frac{\chi \delta \operatorname{Exp}((\alpha+1) \mathrm{h} /(1+\beta \mathrm{h}))}{16(1+\beta \mathrm{h})^{2}}\left(\frac{6}{B \mathrm{i}}+1\right)+ \\
& +9 \cdot\left[\chi \operatorname{Exp}(\alpha \mathrm{h} /(1+\beta \mathrm{h}))-\frac{\delta^{2} \operatorname{Exp}(2 \mathrm{~h} /(1+\beta \mathrm{h}))}{4(\beta \mathrm{h}+1)^{2}}\right]\left(\frac{4}{\mathrm{Bi}}+1\right)+36 \cdot \delta \operatorname{Exp}\left(\mathrm{h} /(1+\beta \mathrm{h})\left(\frac{2}{\mathrm{Bi}}+1\right)\right.
\end{aligned}
$$

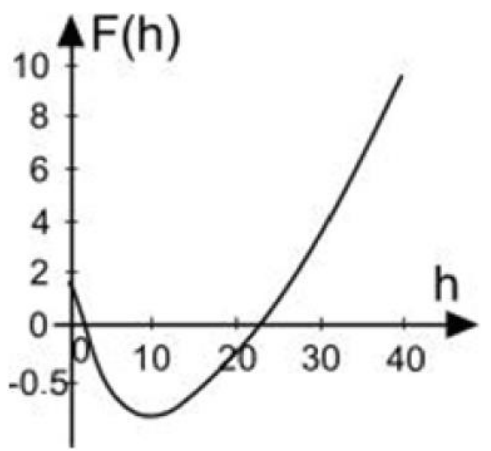

Fig. 1. Graphical representation of the equation with regard to $10 \% \mathrm{~B} 2$ polymethacrylate, taking into account the dissipative and chemical source of heat release under thermal boundary conditions of the first kind for a round pipe. $T_{0}=413 \mathrm{~K}, \mathrm{~T}_{1}=$ $418 \mathrm{~K}$.

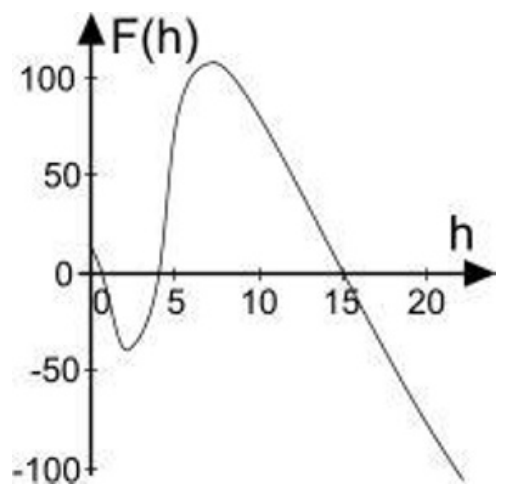

Fig. 2. Graphical representation of the equation with regard to $10 \% \mathrm{~B} 2$ polymetacrylate, taking into account the dissipative and chemical source of heat release under thermal boundary conditions of the third kind for a round pipe. $\mathrm{T}_{0}=413 \mathrm{~K}, \mathrm{~T}_{1}=$ $418 \mathrm{~K}$.
The conducted analysis of behavior of solutions of the characteristic equations for polymetacrylate of grade B2 confirms the existence of critical flow regimes for certain sets of parameters and shows the possibility of switching to the non-calculating operating modes of heat engineering equipment.

\section{Conclusions}

1. A method for analytical investigation has been developed and a theoretical substantiation of the possibility of appearance of bifurcation phenomena in the flow of a nonlinear viscous fluid in an infinite circular pipe under thermal boundary conditions of the first and third kind is presented.

2. A technique for analytical analysis of heat and mass exchange of nonlinear viscous fluids in tubular homophase rectilinear reactors has been developed.

3. When considering the flow of B2 polymetacrylate in a tubular reactor, solutions of characteristic equations were obtained, analysis of which shows the possibility of switching to non-calculating operating modes of heat engineering equipment for certain values of control parameters.

\section{References}

1. S.A. Bostanjinyan, A.G. Merzhanov, S.I. Khudyaev, On the Hydrodynamic Thermal Explosion, Reports of the Academy of Sciences of the USSR, 163(1), 133-136 (1965)

2. V.V. Barzykin, A.G. Merzhanov, The Boundary problem in the thermal explosion theory, Dokl. ANSSSR, 120(6), 1271 (1958)

3. D.M. Clemmov, J.D. Huffingtonn,An extension of the theory of thermal explosion and its application to the oscillatory burning of explosives, Transactions of the Faraday Society, 52, 385-396 (1956)

4. A.G. Merzhanov, Combustion and explosion processes in physical chemistry and technology of inorganic materials, Russ. Chem. Rev., 72(4), 323345 (2003)

5. A.J. Chorin, A numerical method for solving incompressible viscous flow problems, Comput. Phys, 2 (1967)

6. D.O. Glushkov, P.A. Strizhak, Mathematical modeling of heat and mass transfer processes with chemical reaction at polymeric material ignition by several small-size hot particles, Mathematical Problems in Engineering, 2015(614143), 1-8 (2015)

7. S.A. Livshits, Yu. A. Nazmeev, K.M. Malov, Critical regimes of the nonisothermal flow of a viscous fluid in a circular pipe, Fundamental research, 6, 56-57 (2005)

8. R.R. Kantyukov, M.S. Takhaviev, S.A. Livshits, R.V. Lebedev, S.V. Shenkarenko, Solution of the Stationary Equation of Heat Conductivity with the Chemical and Dissipative Heat Source in an Infinite Circular Pipe for a Newtonian Fluid, Vestnik 
Kazanskogo tekhnologicheskogo universiteta, 18(11), 200-205 (2015)

9. R.R. Kantyukov, M.S. Takhaviev, S.A. Livshits, R.V. Lebedev, S.V. Shenkarenko, The Solution of the Stationary Equation of Heat Conductivity with the Chemical Source with the Boundary Thermal Conditions of the Third Kind in an Infinite Circular Pipe, Vestnik Kazanskogo tekhnologicheskogo universiteta, 18(9), 222-225 (2015)

10. R.C. Reid, J.M. Prausnitz, B.E Poling, The properties of gases and liquids, 753 (1987)

11. Y.S. Touloukian, C.Y. Ho, Thermophysical properties of matter (1972)

12. R.V. Lebedev, S.A. Livshits, Investigation of critical flow regimes of generalized viscous media in a tubular reactor, Proceedings of the MAI, 46(1) (2011)

13. S.S. Kutateladze, V.I. Popov, E.M. Khabakhpasheva, Hydrodynamics of liquids with variable viscosity, PMTF, 1, 45-49 (1966)

14. R.R. Kantyukov, M.S. Takhaviev, S.A. Livshits, R.V. Lebedev, S.V. Shenkarenko, Analytical study on the presence of bifurcation phenomena in the flow of nonlinear viscous fluids in channels of complex geometry, Bulletin of the Technological University, 18(4), 223-225 (2015)

15. R.R. Sabitov, S.R. Enikeeva, N.A. Yudina, Using the Pade approximant to determine the ignition conditions of a continuous infinite cylinder filled with a reactive medium under convective heat transfer, Transport and storage of petroleum products and hydrocarbons, 1, 17-21 (2017) 